\title{
The Separation of Two Close Hidden Periodicities
}

\author{
By \\ Michael SHIMSHONI \\ Department of Applied Mathematics, The Weizmann Institute of Science \\ Rehovot, Israel.
}

\section{§1. Introduction}

Let $f(t)$ be the sum of several periodic components of unknown period, amplitudes and phases. We assume that the periods are generally not in a harmonic ratio to each other and we wish to retrieve the unknown periods, amplitudes and phases.

Lanczos [4, pp. 267-272] gives a detailed account of a method of searching for such hidden periodicities. Jeffreys [2] suggests a somewhat modified approach. In two previous papers [5 and 6] I have suggested methods of solving this problem and estimating the uncertainties in the results.

All these procedures use the idea of Fourier Analysis (or Transform) in one way or another. A problem which remained open is that of the resolution in the case of two periods which are "close" to each other. In the present work a method is proposed to handle close periods.

In order to clarify the ideas, let us consider a function $f(t)$ which is the sum of just two harmonic components, i.e.

$$
\begin{aligned}
f(t)= & C_{1} \cos \left(2 \pi t / T_{1}-\varphi_{1}\right) \\
& +C_{2} \cos \left(2 \pi t / T_{2}-\varphi_{2}\right)
\end{aligned}
$$

and let $f(t)$ be given in the interval

$$
-T / 2 \leqslant t \leqslant T / 2 \text {. }
$$

We define as

$$
\sigma_{1}=T / T_{1}, \quad \sigma_{2}=T / T_{2}
$$

which give us the number of full cycles the first or second component, respectively, is contained in our interval of interest. Let $T_{1}<T_{2}$ and thus $\sigma_{1}>\sigma_{2}$.

The direct approach for obtaining $T_{1}, C_{1}$, $\varphi_{1}, T_{2}, C_{2}$ and $\varphi_{2}$ would be to compute the two transform functions

$$
A(s)=\frac{2}{T} \int_{-T / 2}^{T / 2} f(t) \cos \frac{2 \pi s t}{T} d t
$$

$$
B(s)=\frac{2}{T} \int_{-T / 2}^{T / 2} f(t) \sin \frac{2 \pi s t}{T} d t
$$

and from it the functions

$$
\begin{aligned}
& C(s)=\left[A^{2}(s)+B^{2}(s)\right]^{1 / 2} ; \\
& \varphi(s)=\tan ^{-1}[B(s) / A(s)] .
\end{aligned}
$$

The maxima of $C(s)$ should occur close to the values $s=\sigma_{1}$, and $s=\sigma_{2}$, where $\varphi(s) \approx \varphi_{1}$ and $\varphi_{2}$ respectively.

The methods of the above-mentioned authors use this form of transform in somewhat modified forms, but to the following discussion, these variations are of no consequence.

In order to examine the problems arising when $T_{1}$ is close to $T_{2}$, we have to compute the expression (4) and (5) for $f(t)$ given by (1). We get

$$
\begin{aligned}
A(s)= & \frac{C_{1} \cos \varphi_{1} \sin \left[\pi\left(\sigma_{1}-s\right)\right]}{\pi\left(\sigma_{1}-s\right)} \\
& +\frac{C_{2} \cos \varphi_{2} \sin \left[\pi\left(\sigma_{2}-s\right)\right]}{\pi\left(\sigma_{2}-s\right)} \\
& +\frac{C_{1} \cos \varphi_{1} \sin \left[\pi\left(\sigma_{1}+s\right)\right]}{\pi\left(\sigma_{1}+s\right)} \\
& +\frac{C_{2} \cos \varphi_{2} \sin \left[\pi\left(\sigma_{2}+s\right)\right]}{\pi\left(\sigma_{2}+s\right)}, \\
B(s)= & \frac{C_{1} \sin \varphi_{1} \sin \left[\pi\left(\sigma_{1}-s\right)\right]}{\pi\left(\sigma_{1}-s\right)} \\
& +\frac{C_{2} \sin \varphi_{2} \sin \left[\pi\left(\sigma_{2}-s\right)\right]}{\pi\left(\sigma_{2}-s\right)} \\
& -\frac{C_{1} \sin \varphi_{1} \sin \left[\pi\left(\sigma_{1}+s\right)\right]}{\pi\left(\sigma_{1}+s\right)} \\
& -\frac{C_{2} \sin \varphi_{2} \sin \left[\pi\left(\sigma_{2}+s\right)\right]}{\pi\left(\sigma_{2}+s\right)} .
\end{aligned}
$$

Before computing $C(s)$ we shall look at expressions (6.a) and (6.b).

We take $\sigma_{1}$ being close to $\sigma_{2}$ so that

$$
\left(\sigma_{1}-\sigma_{2}\right) /\left(\sigma_{1}+\sigma_{1}\right) \ll 1 \text {. }
$$

The values of $s$ which are of interest are close to $\sigma_{1}$ and $\sigma_{2 .}$. Because of that, the $t w_{0}$ 
later terms of both $A(s)$ and $B(s)$ can be ignored in comparison with their respective two earlier terms. Neglecting these terms will still enable us to get a good idea of the nature of $C(s)$. Under these conditions

$$
\begin{aligned}
C^{2}(s) \approx & \frac{C_{1}^{2} \sin ^{2}\left[\pi\left(\sigma_{1}-s\right)\right]}{\pi^{2}\left(\sigma_{1}-s\right)^{2}}+\frac{C_{2}^{2} \sin ^{2}\left[\pi\left(\sigma_{2}-s\right)\right]}{\pi^{2}\left(\sigma_{2}-s\right)^{2}} \\
& +\frac{2 C_{1} C_{2} \sin \left[\pi\left(\sigma_{1}-s\right)\right] \sin \left[\pi\left(\sigma_{2}-s\right)\right]}{\pi^{2}\left(\sigma_{1}-s\right)\left(\sigma_{2}-s\right)} \\
& \times \cos \left(\varphi_{1}-\varphi_{2}\right) .
\end{aligned}
$$

We see from expression (8) that $C(s)$ is the third side of a triangle, the two other sides of which are

$$
y_{1}=\frac{C_{1} \sin \left[\pi\left(\sigma_{1}-s\right)\right]}{\pi\left(\sigma_{1}-s\right)}
$$

and

$$
y_{2}=\frac{C_{2} \sin \left[\pi\left(\sigma_{2}-s\right)\right]}{\pi\left(\sigma_{2}-s\right)} .
$$

The angle between $y_{1}$ and $y_{2}$ is

$$
\phi=\pi-\left(\varphi_{1}-\varphi_{2}\right) \text {. }
$$

Thus, although near $s=\sigma_{1}, y_{1}$ tends to a maximum, this tendency might be masked by the relatively big $y_{2}$, especially for unfavourable values of $\phi$.

In order to estimate the interference of the period $T_{2}$ with that of $T_{1}$ let us examine the behaviour of the function $S(x)=\frac{\sin \pi x}{\pi x}$ which occurs both in $y_{1}$ and $y_{2}$, defining $S(0)$ $=1$.

As $x$ increases, the maxima of $|S(x)|$ are $.217, .128, .091, .071$, etc., and they occur for values of $x=1.43,2.46,3.47,4.48$, etc. The maxima decrease almost proportionately to $x^{-1}$.

If $\sigma_{1}-\sigma_{2}>5$ the interference of the second component with the first is less than $6 \%$ (assuming $C_{1} \approx C_{2}$ ). On the other hand, if $\sigma_{1}-\sigma_{2}<2$, we may have interference which exceeds $20 \%$, and for $\sigma_{1}-\sigma_{2}<1$ the situation may be so bad that the maxima in $C(s)$ at $s=\sigma_{1}$ caused by $y_{1}$ will be so disturbed by $C_{2} S\left(\sigma_{2}-s\right)$, that in $C(s)$ no maximum will be found near enough to $s=\sigma_{1}$ to help discover the " hidden" period $T_{1}$.

Lanczos $[4$, p. 271], when dealing with close periods, suggests a weighting method which decreases the extreme values of the side lobes faster than in the function $S(x)$, but his method is only useful for cases of $\sigma_{1}-\sigma_{2}>1$; if $\sigma_{1}-$ $\sigma_{2}<1$ his modification makes the separation even more difficult, because his corresponding $S(x)$ is "somewhat broader" than ours, in particular for small $x$.

From the above discussion one should not conclude that, from analysing expression (8), we hoped to obtain the exact values of $\sigma_{1}$ and $\sigma_{2}$, their amplitudes and their phases. All we need is a "good" approximation of either $\sigma_{1}, C_{1}$ and $\varphi_{1}$ or $\sigma_{2}, C_{2}$ and $\varphi_{2}$. Such an approximation, say, $\bar{\sigma}_{1}, \bar{C}_{1}$ and $\bar{\varphi}_{1}$, is considered "good", if, when we form by subtraction the new function

$$
f^{1}(t)=f(t)-\bar{C}_{1} \cos \left(2 \pi \bar{\sigma}_{1} t / T-\bar{\varphi}_{1}\right),
$$

the resulting $f^{1}(t)$ when analysed will yield $\sigma_{2}, C_{2}$ and $\varphi_{2}$ more easily than $f(t)$. In other words we want

$$
C_{1} \cos \left(2 \pi \sigma_{1} t / T-\varphi_{1}\right)-\bar{C}_{1} \cos \left(2 \pi \bar{\sigma}_{1} t / T-\bar{\varphi}_{1}\right)
$$

to interfere less with

$$
C_{2} \cos \left(2 \pi \sigma_{2} t / T-\varphi_{2}\right)
$$

than does

$$
C_{1} \cos \left(2 \pi \sigma_{1} t / T-\varphi_{1}\right)
$$

by itself. If this condition is met, we can then get a "good" approximation $\bar{C}_{2}, \bar{\sigma}_{2}, \bar{\varphi}_{2}$ of the second periodicity which, when subtracted from $f(t)$ of expression (1), will enable us to get a still better approximation of the first periodicity. Iteration of this process then leads to convergence.

If we fail to obtain a "good" approximation in the above sense, there is little hope to separate the two periodicities.

In order to illustrate the behaviour of $C(s)$ for various pairs of $\sigma_{1}, \sigma_{2}$ and $\varphi_{1}, \varphi_{2}$ we show in Fig. 1 the dependence of $C(s)$ on $s$ for several typical cases. In all examples I took $C_{1}=C_{2}=1$ because if $\left|C_{1}\right|>\left|C_{2}\right|$ it is easier to obtain a good approximation to the first periodicity than in the case of equal $C_{1}$ and $C_{2}$. As only the difference $\varphi_{1}-\varphi_{2}$ appears in expression (8), I put $\varphi_{2}=0$ and let $\varphi_{1}$ vary between 0 and $\pi$. $T_{2}$ is taken as 157 seconds 

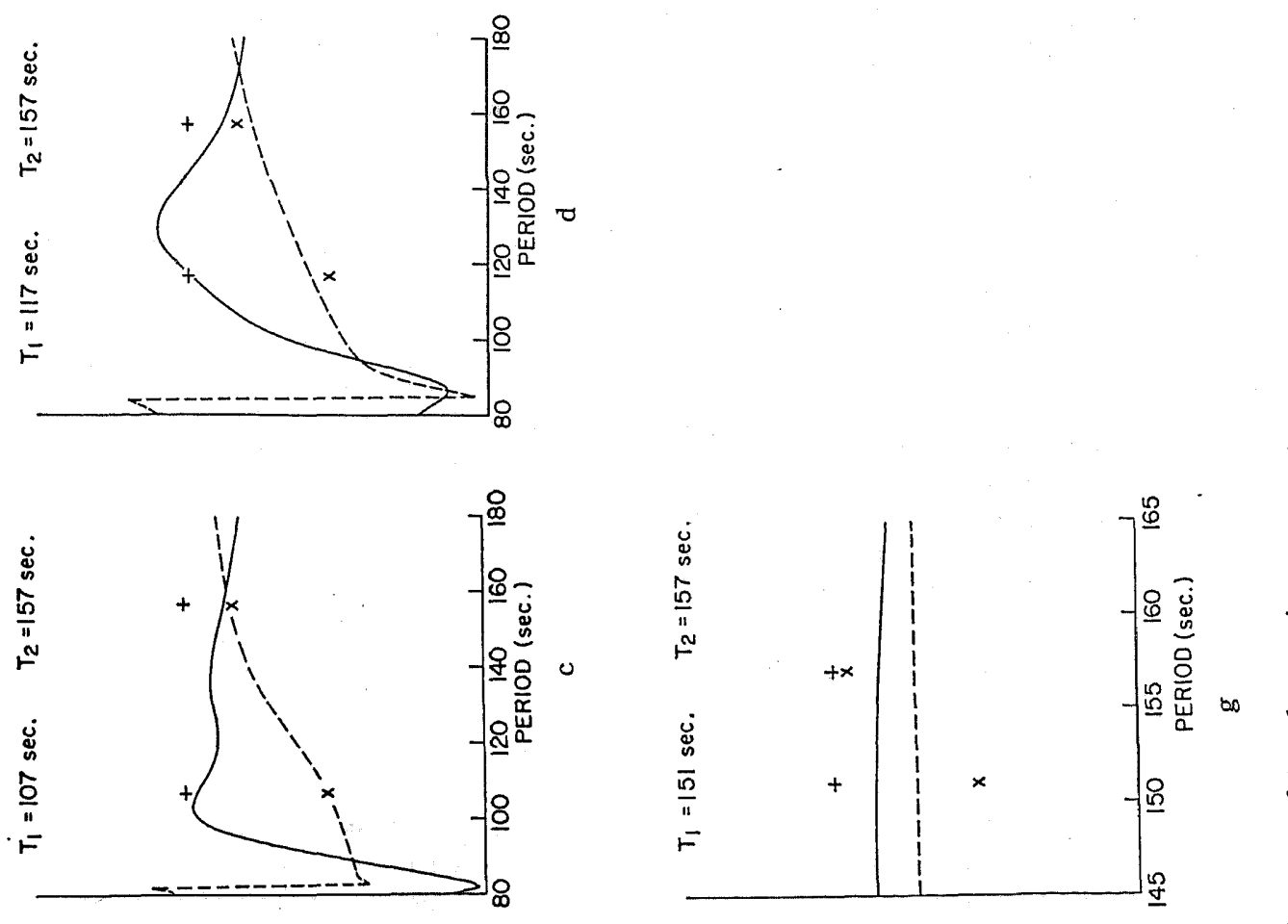

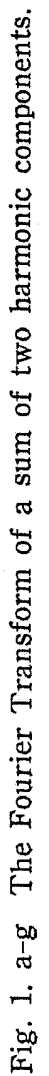
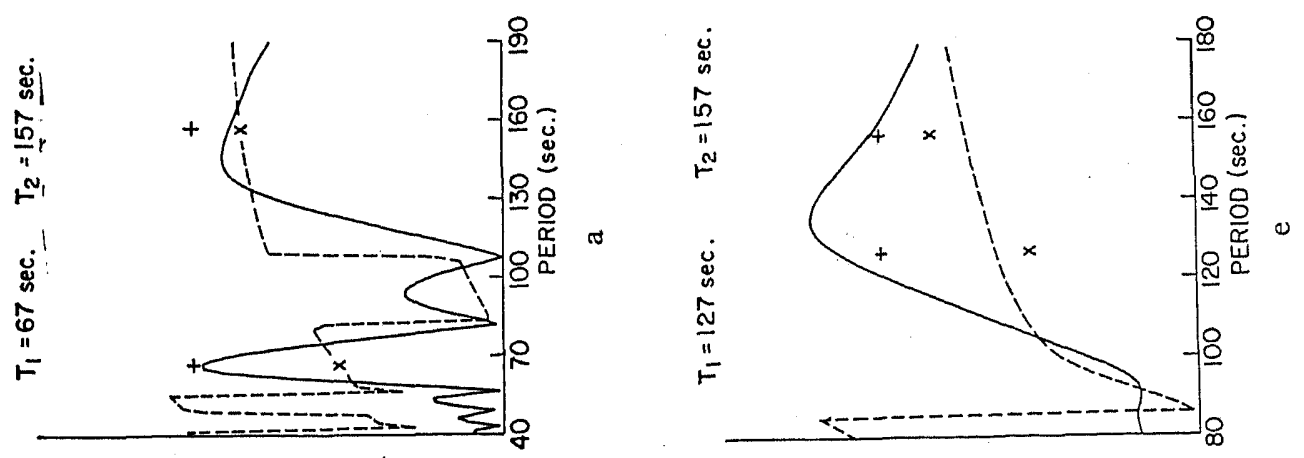
and $T_{1}$ varies. The length of the sample is always 360 seconds, the sampling interval is 1 second.

The continuous line in the figures plots the amplitude $C(T)$, the broken line plots the phase $\varphi(T)$. The two + signs show $C_{1}\left(T_{1}\right)$ and $C_{2}\left(T_{2}\right)$ respectively and the two $\times$ signs indicate $\varphi_{1}\left(T_{1}\right)$ and $\varphi_{2}\left(T_{2}\right)$. In the ideal case the continuous line should have two maxima through the + signs, and the broken line should go through the $x$.

We see in Figs. 1a, $1 \mathrm{~b}$ and $1 \mathrm{c}$ that for $T_{1}$ $=67,87$, and 107 seconds, separation is quite good. For $T_{1}=107, \sigma_{1}-\sigma_{2}=1.1$ and this seems to suffice for separation. I tried it for several values of $\varphi_{1}$ and separation was possible in most cases.

For $T_{1}>107$ seconds separation becomes increasingly more difficult. Figs. $1 \mathrm{~d}$ and le show $T_{1}=117$ and 127 respectively. In both cases it is impossible to pick out $T_{1}$ or $T_{2}$ as maxima of the continuous line.

Figs. If and $1 \mathrm{~g}$ treat the case $T_{1}=151\left(\sigma_{1}-\right.$ $\sigma_{2}=.09$ ) for two typical values of $\varphi_{2}$. It is quite clear from these figures why separation under such circumstances is so difficult.

\section{§2. The Method of Separation}

Let $f(t)$ be as defined in expression (1). We shall define two other functions, an even function

$$
e(t)=\frac{1}{2}[f(t)+f(-t)]
$$

and an odd function

$$
o(t)=\frac{1}{2}[f(t)-f(-t)] .
$$

Both $e(t)$ and $o(t)$ are defined for the interval (2), namely

$$
-T / 2 \leqslant t \leqslant T / 2
$$

In the following discussion we shall concentrate on $e(t)$, but with minor obvious changes the analysis can be applied to $o(t)$ as well.

Substituting expression (1) into (11), we find

$$
\begin{aligned}
e(t)= & {\left[C_{1} \cos \varphi_{1}\right] \cos \left(2 \pi t / T_{1}\right) } \\
& +\left[C_{2} \cos \varphi_{2}\right] \cos \left(2 \pi t / T_{2}\right)
\end{aligned}
$$

Instead of attempting to determine $T_{1}$ and $T_{2}$ (or $\sigma_{1}$ and $\sigma_{2}$ ) from $f(t)$, we shall do it using $e(t)$ as given in expression (12). We see that $e(t)$ is the sum of two pure cosine terms (there is no phase angle) of periods $T_{1}$ and $T_{2}$ with the amplitudes $A_{1}=C_{1} \cos \varphi_{1}$ and $A_{2}=C_{2} \cos \varphi_{2}$. Separation of the two terms will depend, as stated before, on the ratio

$$
\rho=A_{1} / A_{2}=\left(C_{1} / C_{2}\right)\left(\cos \varphi_{1} / \cos \varphi_{2}\right) .
$$

If $|\rho| \gg 1,\left|A_{1}\right| \gg\left|A_{2}\right|$ and it is relatively easy to determine $A_{1}$ as $A_{2}$ will only disturb in an insignificant way. We should be able to

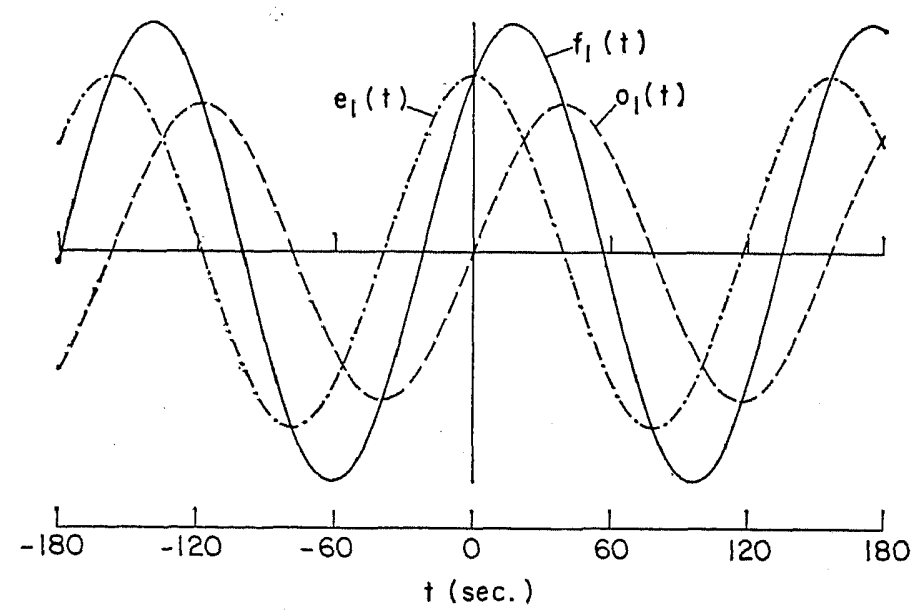

Fig. 2 The separation of a simple harmonic function into odd and even components. 
obtain a "good" estimate of $T_{1}$. Conversely, if $|\rho| \ll 1$, a "good" estimate of $T_{2}$ can be obtained.

In order to illustrate how $\rho$ can be altered at will, let us look at Fig. 2 which shows one of the components of $f(t)$, namely

$$
\begin{aligned}
f_{1}(t)= & C_{1} \cos \left(2 \pi t / T_{1}-\varphi_{1}\right) \\
& =C_{1} \cos \left[2 \pi\left(t-\varphi^{\prime}{ }_{1}\right) / T_{1}\right],
\end{aligned}
$$

where

$$
\dot{\varphi}^{\prime}{ }_{1}=T_{1} \varphi_{1} / 2 \pi
$$

is the rescaled phase angle.

In addition, the two functions

$$
e_{1}(t)=\left(C_{1} \cos \varphi_{1}\right) \cos 2 \pi t / T_{1}
$$

and

$$
o_{1}(t)=\left(C_{1} \sin \varphi_{1}\right) \sin 2 \pi t / T_{1}
$$

have been drawn in Fig. 2. These two functions are the $T_{1}$ components of $e(t)$ and $o(t)$. As stated before, our attention is concentrated at the moment on $e_{1}(t)$, but we notice that

$$
f_{1}(t)=e_{1}(t)+o_{1}(t) .
$$

We shall now change our time origin by $\tau$ and look at the function

$$
\begin{aligned}
\bar{f}_{1}(t) & =f_{1}(t-\tau)=C_{1}\left[\cos 2 \pi(t-\tau) / T_{1}-\varphi_{1}\right] \\
& =C_{1}\left[\cos 2 \pi\left(t-\bar{\varphi}^{\prime}{ }_{1}\right) / T_{1}\right],
\end{aligned}
$$

where

$$
\bar{\varphi}_{1}^{\prime}=\varphi_{1}^{\prime}+\tau=T_{1} \varphi_{1} / 2 \pi+\tau .
$$

If we now split $\bar{f}_{1}(t)$ into an even function $\bar{e}_{1}(t)$ and an odd function $\bar{o}_{1}(t)$, the amplitude of $\bar{e}_{1}(t)$ will be

$$
\begin{aligned}
\bar{A}_{1}(\tau)= & C_{1} \cos \left(2 \pi \bar{\varphi}_{1}^{\prime} / T_{1}\right) \\
& =C_{1} \cos \left(\varphi_{1}+2 \pi \tau / T_{1}\right) .
\end{aligned}
$$

Examining $\bar{A}_{1}(\tau)$ in expression (21) we see its dependence on $\tau$; by varying $\tau,\left|\bar{A}_{1}(\tau)\right|$ can take any value between $C_{1}$ and zero.

In Fig. $3, \tau$ is so chosen that $\bar{A}_{1}(\tau)$ is rather small; by a slight further shift of $\tau$ we could make $\bar{A}_{1}(\tau)$ disappear altogether.

By choosing $\tau$ so that $\bar{A}_{1}(\tau)$ almost, or completely, vanishes, we can make $|\rho| \ll 1$. Thus for such values of $\tau$ it will be easy to determine $T_{2}$ with great accuracy. Inspecting Fig. 2 again we see that if the origin is shifted to places where $f_{1}(t)$ is zero, we have a value of $\tau$ which will enable us to reduce the presence of $\bar{e}_{1}(t)$. If we shift the origin to places where $f_{1}(t)$ has a maximum or a minimum, we get values of $\tau$ where $\bar{o}(t)$ has no $T_{1}$ component, because in these places $\bar{o}_{1}(t)=0$.

It should be realized that by a time shift $\tau$, not only is $A_{1}$ changed to $\bar{A}_{1}(\tau)$, but also $A_{2}$ (or the period $T_{2}$ ) changes to $\bar{A}_{2}(\tau)$. We can write the ratio $\rho(\tau)$ as function of $\tau$ :

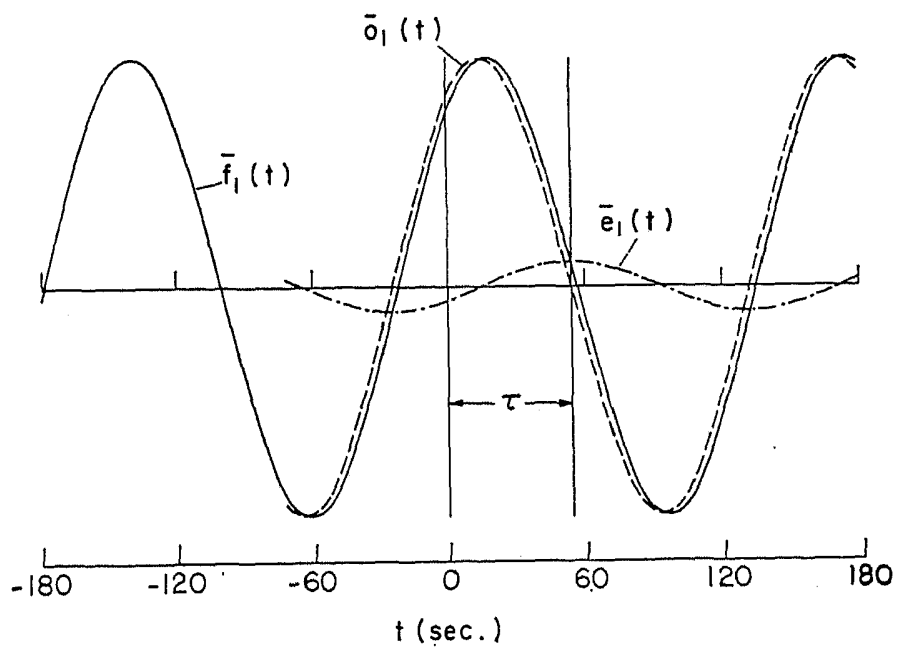

Fig. 3 The effect of shifting the time origin, on the separation of a simple harmonic function into odd and even components. 


$$
\begin{aligned}
\rho(\tau)= & \bar{A}_{1}(\tau) / \bar{A}_{2}(\tau)=\left(C_{1} / C_{2}\right)\left[\operatorname { c o s } \left(\varphi_{1}\right.\right. \\
& \left.\left.+2 \pi \tau / T_{1}\right) / \cos \left(\varphi_{2}+2 \pi \tau / T_{2}\right)\right] .
\end{aligned}
$$

Inspecting $\rho(\tau)$ we see that in it we have the ratio of two cosine functions of different periods, which will not always vanish at the same moment. So we will have values of $\tau$ for which $|\rho(\tau)| \ll 1$ and also some for which $|\rho(\tau)| \gg 1$.

Unfortunately, we have been putting the cart before the horse. We have no direct method of choosing $\tau$ so that $\overline{A_{1}}(\tau)$ of expression (21) is zero, since this would imply knowledge of $T_{1}$ and $\varphi_{1}$, two of the quantities we are trying to find.

In the following, I suggest a procedure to find values of $\tau$ which make $\rho(\tau)$ tend either to infinity (yielding $T_{1}$ ) or to zero (yielding $T_{2}$ ).

For any value of $\tau$ we try to find the best approximation to $\bar{e}(t)$ in the least squares sense, by a single, even periodic function

$$
A \cos \left(2 \pi t / T_{0}\right) \text {. }
$$

As $\bar{e}(t)$ is an even function it is defined only in the interval

$$
-\bar{I} / 2 \leqslant t \leqslant \bar{T} / 2
$$

where

$$
\bar{T} / 2=T / 2-|\tau| \text {. }
$$

We must also remember that $\bar{e}(t)$ is a function of $\tau$. We define the positive normalised deviation function

$$
\begin{aligned}
R(\tau, T)= & \int_{-\bar{i} / 2}^{\bar{T} / 2}\left[\bar{e}(t)-A \cos \left(2 \pi t / T_{0}\right)\right]^{2} d t \\
& / \int_{-\bar{T} / 2}^{\bar{T} / 2}[\bar{e}(t)]^{2} d t
\end{aligned}
$$

and for a fixed $\tau$ we find a value of $T_{0}=T_{\tau}$ such that $R\left(\tau, T_{\tau}\right)$ is minimal.

This process is repeated for many values of $\tau$, and we now look for minima among the various $R\left(\tau, T_{\tau}\right)$ for the different $\tau$. It is clear that if $\tau$ is a value for which $|\rho(\tau)|$ $\rightarrow \infty, R\left(\tau, T_{\tau}\right)$ will be conspicuous by being zero and $T_{\tau}=T_{1}$. In analogy, if $\tau$ is a value such that $|\rho(\tau)| \rightarrow 0$, we shall find $T_{\tau}=T_{2}$.

\section{§3. The Method in Practice}

In practical cases we shall have $f(t)$ for discrete values of $t$ only. We shall define, in analogy with expression (1),

$$
\begin{aligned}
f_{r}= & C_{1} \cos \left(2 \pi r / T_{1}-\varphi_{1}\right) \\
& +C_{2} \cos \left(2 \pi r / T_{2}-\varphi_{2}\right)
\end{aligned}
$$

for $-n \leqslant r \leqslant n$. Without loss of generality, I have re-scaled the time so that the time interval between adjacent points is unity.

In analogy with the expressions for continuous $t$ we can define $e_{r}$ and $\bar{e}_{r}$. For simplicity we shall choose integral values for $\tau$, although it is not very difficult to handle non-integral $\tau$ as well.

We have now

$$
\begin{aligned}
\bar{e}_{r}= & \left\{C_{1} \cos \left(\varphi_{1}+2 \pi \tau / T_{1}\right)\right\} \cos \left(2 \pi r / T_{1}\right) \\
& +\left\{C_{2} \cos \left(\varphi_{2}+2 \pi \tau / T_{2}\right)\right\} \cos \left(2 \pi r / T_{2}\right)
\end{aligned}
$$

defined for values of $r$ in the interval

$$
-\bar{n} \leqslant r \leqslant \bar{n},
$$

where

$$
\bar{n}=n-|\tau| \text {. }
$$

We define the normalised deviation function by the quotient of two sums,

$$
R(\tau, T)=\sum_{r=-\bar{n}}^{\bar{n}}\left[\bar{e}_{r}-A \cos \left(2 \pi r / T_{0}\right)\right]^{2} / \sum_{r=-\bar{n}}^{n} \bar{e}_{r}^{2} .
$$

We replaced integration by ordinary summation, and not by an interpolation-summation procedure as suggested by Filon [1] and others. Not only do these interpolation procedures not improve accuracy in cases of harmonic analysis, but they actually give a biased result depending on the period. Jeffreys and Jeffreys [3, p. 456] show this to be so in the case of linear interpolation, but a bias is caused with higher order interpolation formulae as well.

In the Appendix I give explicit formulae for the evaluation of expression (29).

For each value of $\tau$ we find $T_{0}=T_{\tau}$ so that $R\left(\tau, T_{\tau}\right)$ is minimal. For all the values of $\tau$ we use, we sort the $R\left(\tau, T_{\tau}\right)$ obtained in increasing order of $T_{\tau}$, retaining the relevant $A\left(=A_{\tau}\right)$ as well. We inspect the list of $R\left(\tau, T_{\tau}\right)$ for small values and these should occur for $T_{\tau} \simeq T_{1}$ or $T_{2}$ giving

$$
A \tau \simeq C_{1} \cos \left(\varphi_{1}+2 \pi \tau / T_{1}\right)
$$

or

$$
A \tau \simeq C_{2} \cos \left(\varphi_{2}+2 \pi \tau / T_{2}\right)
$$


respectively.

Thus we have an approximation to $T_{1}$ and $C_{1} \cos \left(\varphi_{1}+2 \pi \tau / T_{1}\right)$ (or $T_{2}$ and $C_{2} \cos \left(\varphi_{2}+2 \pi \tau\right)$ $\left.T_{2}\right)$ ). Analysis of the odd function would yield approximations to $C_{1} \sin \left(\varphi_{1}+2 \pi \tau / T_{1}\right)$ or $C_{2} \sin \left(\varphi_{2}+2 \pi \tau / T_{2}\right)$ as well, enabling us to separate $C_{1}$ from $\varphi_{1}$ or $C_{2}$ from $\varphi_{2}$.

\section{§4. Examples}

One of the test cases I analysed has the values $T_{1}=151$ seconds, $T_{2}=157$ seconds; $C_{1}$
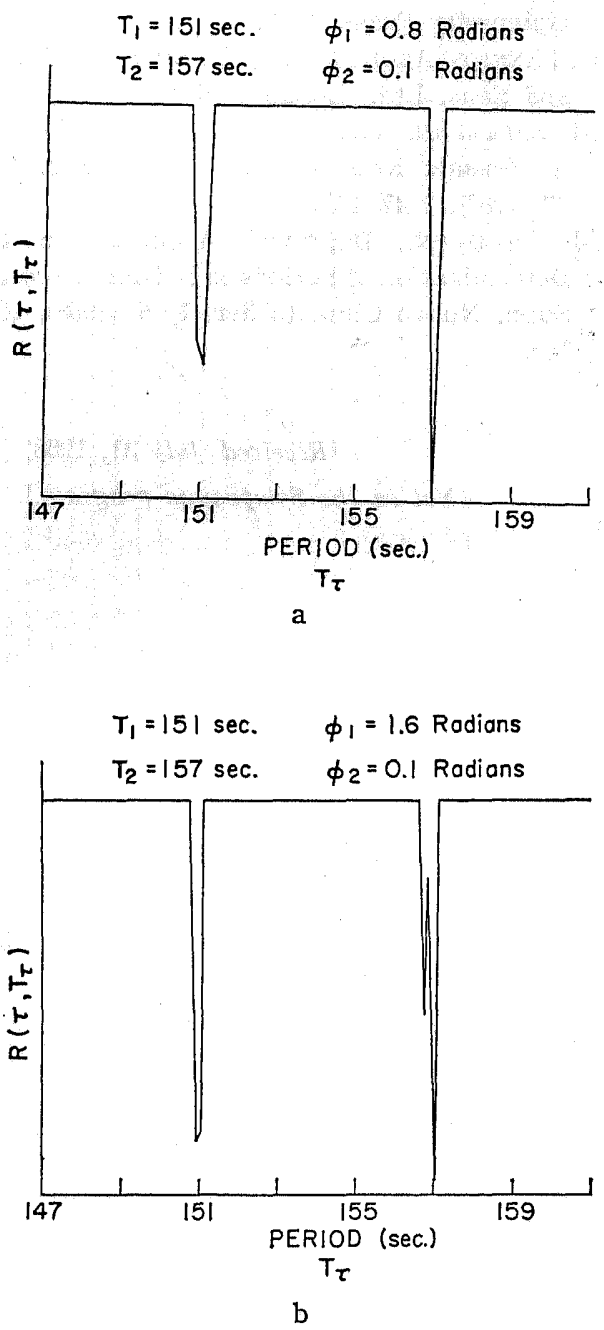

Fig. $4 a-4 b$ The minima of the normalised deviation function of a sum of two harmonic components.
$=C_{2}=1, \varphi_{2}=.1$ Radians, and I examined various values of $\varphi_{1}$. I shall report here on two typical cases: $\varphi_{1}=.8$ Radians and $\varphi_{1}=$ 1.6 Radians. In all the cases, 361 points at 1-second intervals were taken, making the total length of consideration 360 second.

We had $\sigma_{1}=360 / 151=2.38$ and $\sigma_{2}=2.29$; thus the change in phase between the two components over the whole length of the sample was about $33^{\circ}$. In Figs. $4 \mathrm{a}$ and $4 \mathrm{~b}$ the behaviour of the deviation function $R\left(\tau, T_{\tau}\right)$ is given. $R\left(\tau, T_{\tau}\right)$ is the minimum value of $R\left(\tau, T_{0}\right)$ for a fixed $\tau$. The value of $T_{0}$ for which $R\left(\tau, T_{0}\right)$ is minimum is called $T_{\tau}$, and $T_{\tau}$ is the abscissa in Figs. $4 \mathrm{a}$ and $4 \mathrm{~b}$. In Figs. $4 \mathrm{a}$ and $4 \mathrm{~b}$ the horizontal portions of the lines indicate that there $R\left(\tau, T_{\tau}\right)$ was so great that it was necessary to truncate it so that the scale of the figure remained meaningful. Actually at these truncated sections, the function took values several orders of magnitude bigger than for the minimal points. A comparison of Figs. $4 \mathrm{a}$ and $4 \mathrm{~b}$ with Figs. If and $1 \mathrm{~g}$ indicates the power of the present method.

From these two figures, we see that one can get quite readily very good approximations to $T_{1}$ and $T_{2}$. We show only the analysis of $\bar{e}(t)$, but comparable results were obtained using $\bar{o}(t)$. From our results we obtained very good estimates of the amplitudes and phases as well, even without iterating the process in the sense of expression (10). If one iteration is added, convergence was complete in a numerical sense.

\section{§5. Acknowledgement}

This research was supported by the U.S. Office of Naval Research under contract N00014-67-C0146.

\section{Appendix}

The Approximation of $\bar{e}(t)$ by $A \cos 2 \pi t / T_{0}$.

We shall consider values of $\bar{e}(t)$ for discrete values of $t$, and use the notation $\bar{e}_{r}$ for $\bar{e}(r)$. We are trying to minimise $R\left(\tau, T_{0}\right)$ as given in Eq. (29), by choosing the best values of $A$ and $T_{0}$.

To find these $A$ and $T_{0}$ we have to solve 


$$
\frac{\partial R\left(\tau, T_{0}\right)}{\partial T_{0}}=0
$$

and

$$
\frac{\partial R\left(\tau, T_{0}\right)}{\partial A}=0 .
$$

From $(A 2)$ we get

$$
-\Sigma \bar{e}_{r} \cos \left(2 \pi r / T_{0}\right)+A \Sigma \cos ^{2}\left(2 \pi r / T_{0}\right)=0
$$

or

$$
A=\frac{\sum \bar{e}_{r} \cos \left(2 \pi r / T_{0}\right)}{\frac{1}{2}\left\{2 \bar{n}+1+\frac{\sin \left[(2 \bar{n}+1) 2 \pi / T_{0}\right]}{\sin \left(2 \pi / T_{0}\right)}\right\}} .
$$

All the summations are from $r=-\bar{n}$ to $r=\bar{n}$.

We can substitute the value of $A$ from (A4) into (29) and, after some simple manipulations we have

$$
\begin{aligned}
R\left(T_{0}\right) & =1 \\
& -\frac{\left[\sum \bar{e}_{r} \cos \left(2 \pi r / T_{0}\right)\right]^{2}}{\frac{1}{2}\left\{2 \bar{n}+1+\frac{\sin \left[2 \pi(2 \bar{n}+1) / T_{0}\right]}{\sin \left(2 \pi / T_{0}\right)}\right\} \sum \bar{e}_{r}^{2}} .
\end{aligned}
$$

Unfortunately equation (A1) is unsolvable analytically; thus we have to look for minima in (A5) numerically. This can be done by a modified Newton-Rapson procedure.

\section{References}

1. L. N. G. FiLON, On a Quadrature Formula for Trigonometric Integrals, Proc. Roy. Soc. Edin. 49 (1928), 38-47.

2. H. Jefrreys, Note on Fourier Analysis, Bull. Seism. Soc. Am. 54 (1964), 1441-1444.

3. H. Jefrreys and B. S. Jefrreys, Methods of Mathematical Physics, 3rd ed., Cambridge University Press, 1956.

4. C. Lanczos, Applied Analysis, Sir Isaac Pitman and Sons, Ltd., London, 1964.

5. M. Shimshoni, The Determination of Periods in Seismic Records, Bull. Seism. Soc. Am. 57 (1967), 1347-1354.

6. M. SHIMSHoni, Improved Accuracy in the Determination of Periods in Seismic Records, Supp. Nuovo Cimento Ser. I, 6 (1968), 160165.

(Received July 31, 1968)

(Not in the Symposium program.) 